\title{
Approche interculturelle et enseignement des langues à des scientifiques
}

Rapide analyse linguistique

\section{Evelyne Brouzeng}

\section{OpenEdition \\ Journals}

Édition électronique

URL : http://journals.openedition.org/asp/4369

DOI : 10.4000/asp.4369

ISSN : 2108-6354

\section{Éditeur}

Groupe d'étude et de recherche en anglais de spécialité

Édition imprimée

Date de publication : 1 mars 1993

Pagination : 189-207

ISSN : 1246-8185

Référence électronique

Evelyne Brouzeng, «Approche interculturelle et enseignement des langues à des scientifiques », ASp

[En ligne], 1 | 1993, mis en ligne le 26 avril 2014, consulté le 04 mai 2019. URL : http://

journals.openedition.org/asp/4369; DOI : 10.4000/asp.4369

Ce document a été généré automatiquement le 4 mai 2019.

Tous droits réservés 


\title{
Approche interculturelle et enseignement des langues à des scientifiques
}

Rapide analyse linguistique

\author{
Evelyne Brouzeng
}

1 Enseignement de langue de spécialités, English for specific purposes, enseignement de la communication à des scientifiques, autant de courants, d'écoles que sous-tendent des théories pédagogiques et culturelles distinctes. Envisager la distinction entre ces dénominations ne relève pas seulement d'une simple analyse linguistique, mais permettra de décrire des pratiques différentes qui font (les unes aussi bien que les autres) autorité.

2 Le concept de langue de spécialité correspond à une description (un temps opératoire) d'un développement stratifié et cloisonné des disciplines (l'électronique, la mécanique...) on peut légitimement se demander si cette représentation ne remonterait pas à l' Encyclopédie de Diderot sans avoir été réévaluée depuis.

3 Aujourd'hui, c'est l'évidence, ce schéma ne fonctionne plus, de nouvelles technologies se sont développées (l'informatique, l'électronique, « enabling technologies » disent les Anglosaxons des technologies de base de service en somme).

4 Simultanément, l'automatisation, la robotisation, l'informatique industrielle (controls) ont profondément modifié le procès de production.

5 Les frontières entre les disciplines se sont estompées, désormais, elles sont floues, mouvantes - voire totalement artificielles. De nombreux Polytechnics britanniques, conscients du caractère artificiel de ces découpages ont récemment regroupé leurs départements de Génie mécanique et d'électronique.

6 En outre, après avoir prôné la double compétence (électronique + biologie ou mécanique + génie civil, etc.) les chasseurs de têtes des entreprises reconnaissent aujourd'hui les vertus « des généralistes ». 
7 Du point de vue sociologique, l'anglais de spécialité s'adressait de façon privilégiée aux apprenants (en formation initiale ou continue) des filières techniques issus de ce qui fût, en un temps pas si lointain, désigné comme les Écoles pratiques avant de devenir des lycées professionnels ou techniques. À partir de 1966-67, la création des IUT a fait apparaître un nouveau type de technicien, issus pour partie des filières classiques (C et D). À cela il faut ajouter les élèves des écoles d'ingénieurs.

8 La progression numérique, dans le tissu industriel, de cette population active est spectaculaire ils étaient, toutes catégories confondues, moins de 900000 en 1954, ils sont aujourd'hui quatre millions (INSEE 1988). Le contenu de leur fonction s'est rapidement transformé (Stoffaes 1981).

9 L'introduction de nouveaux outils (dans la production, mais aussi dans les services) requiert une part sans cesse croissante d'activité intellectuelle. La nécessité de gérer de l'information en anglais est rapidement apparue (messages d'erreur sur ordinateur, notices d'utilisation des matériels, instructions de maintenance). D'où la réponse très ancrée dans le « métier » (l'anglais pour la secrétaire, la standardiste, le garagiste...).

Une étude de la représentation des métiers dans nos matériaux pédagogiques reste à faire, s'attacher à y analyser l'image qui y est donnée du rôle des femmes ne manquerait pas d'intérêt - leur place dans la production par exemple si Business Objectives de V. Hollett (1991) à la volonté de donner une place aux femmes dans l'entreprise (p. 8) à travers l'organigramme, dans le même temps l'iconographie contredit ce bel optimisme et les filles sont totalement absentes des photos d'ateliers et de laboratoires ( $\mathrm{p} 25,40,41$, 125, 134, 135). D’une façon générale, les métiers des filles sont ceux de secrétariat, de relations publiques.

\section{Les enseignants de langue de spécialité ont-ils choisi le modèle d'Hermès ?}

11 Hermès est à la fois, dieu des voleurs (a-t-il vraiment volé le cheval d'Apollon ?) et de la communication interne, Dieu des routes et des chemins et externe, messager de Zeus (Jacques 1979 : 218).

12 La communication sur « le modèle d'Hermès » vise selon F. Jacques à reproduire l'idéal du vendeur : quoi dire, à qui à quel effet ? Est la copie de quoi vendre, à qui, avec quel effet ?

13 Parler d'une langue "for specific purposes » opère, il est vrai, une distinction voire une hiérarchisation entre diverses activités langagières et les situations dans lesquelles elles se déroulent : to welcome an overseas visitor, to choose between different suppliers, to arrange a visit. Ce découpage trouve son intérêt pédagogique dans un processus de production " assistée ", " guidée ", mais qui ne peut qu'être une étape vers autre chose. Cette " autre chose » mérite de retenir notre attention.

L'ordonnancement du monde par rapport à un individu «invariant», le technicien, électronicien ou informaticien ou le cadre chargé de la commercialisation d'un produit ne peut que constituer un point de référence temporaire et non une finalité.

Quelle est par exemple la signification de «minimum competence» ou de «lower Business English»? Qui concerne-t-elle? En fonction de quelles normes? De quelles représentations? Pour quoi faire? 
Ce libellé porte en lui ses limites (lower, minimum, intermediate), il peut paraître réducteur en regard des besoins tels qu'ils sont ressentis par certains apprenants ou au contraire trop ambitieux pour répondre à d'autres besoins (cas des techniciens du CPA).

Est récemment apparu comme venant se substituer (ou se fondre) au concept « English for specific purposes» celui de "business and technical communication $»^{1}$, dont le champ d'investigation recouvre aussi bien la pédagogie de l'écrit technique (rapport, article) que des aspects plus nouveaux comme "l'écrit sur le lieu de travail », " écrire en groupe », la culture d'entreprise, la communication et l'encadrement, les premiers travaux semblent se consacrer davantage à l'écrit qu'à la communication orale. Jusqu'à présent le corpus des écrits de la communication interne a été étudié (note de service rapport, etc.).

Il semble que la culture de référence soit la culture "managériale », ou/et la culture d'entreprise. L'absence de travaux sur l'oral montre bien la difficulté de sortir du modèle de la « communication marchande » qui vise à séduire (efficacité des textes, utilisation des images et des diagrammes) convaincre (présentation des matériels par ex de leurs caractéristiques et performances).

9 La référence aux contenus dans cette session m'oblige à reconnaître que si l'on considère avec F. Jacques qu'une «technique de communication ne vaut que par le degré de dialogisme qu'elle permet ou dont elle est investie», nous devons beaucoup nous intéresser aux processus à mettre en place pour sortir des productions « manipulées ».

Ces processus doivent nous permettre de passer d'une situation d'accompagnement à une situation d'autonomie (free practice) l'essence de l'autonomie étant ses «degrés de liberté » (unpredictability)

\section{La référenciation au réel}

«La vie du langage est par essence un parler et un dire ; comme parler il est une activité communicative, comme dire il est une activité de référenciation au réel » (Jacques 1979). Attachons-nous à ce lien incontournable avec le réel. Nous avons vu que nos apprenants seront (ou sont) des techniciens, des ingénieurs des entreprises de production et de service.

2 Des changements profonds sont intervenus dans nos sociétés techniciennes (autour de 1973 aux USA vers les années 1980 chez nous). Nous avons rapidement évoqué l'évolution quantitative de ces catégories (ce qui nous place devant des responsabilités de formation que nous ne pouvons pas toujours assumer faute de moyens et de nombreuses filières universitaires ne proposent pas de langues à leurs étudiants).

23 Examinons l'évolution «qualitative» des mutations pour tenter de comprendre l'émergence des nouveaux besoins telle qu'elle se manifeste chez les futurs acteurs. Nous nous efforcerons d'en dégager les enjeux pour la formation.

\section{Du taylorisme pur et dur à des formes nouvelles de gestion des hommes}

24 En avril 1982 lors du premier Colloque de l'AFCERQ (Association française des cercles de qualité), M. Crozier de l'Institut de l'entreprise note :

la vision nouvelle d'une entreprise allégée, formée au commandement ouvert et capable de faire coopérer plus facilement un état-major alerte, vigoureux, beaucoup mieux informé, et des exécutants beaucoup plus qualifiés et beaucoup plus 
responsables est bien sûr très séduisante. Mais cette entreprise n'a plus besoin d'autant de cadres. Ceux dont elle aura besoin devront être différents. Les cadres et la maîtrise vont connaître une mutation difficile. La hiérarchie se transforme, leur rôle se modifie. Ils étaient officiers ou sous-officiers. Ils se réveillent animateurs ou spécialistes [...]. (Crozier 1977)

Ainsi, le post-taylorisme oblige à une nécessaire redéfinition du rôle de l'encadrement et des opérateurs, la mise en place volontariste de la part des directions d'entreprise de la gestion participative fait apparaître des structures de gestion de l'information et de la communication (cercle de Qualité, groupe de progrès), ces réponses à la volonté des salariés de "travailler autrement » ont - dit-on alors - fait leurs preuves au Japon (à partir de 1950 et aux USA dès 1975).

Ces changements concernent les ateliers de production et de service et donc les travailleurs manuels ceux auxquels on ne demandait pas de penser, mais de produire.

Il faut, dit H. Serieyx Directeur général d'Eurequip et conseiller du Commerce extérieur, "mobiliser l'intelligence de l'entreprise» (1982) et La théorie Z de Ouchi (1980) décrit l'organisation des entreprises qui accroissent leur compétitivité en permettant à chacun d'exprimer sa créativité. En France, M. Albert, Ancien Commissaire au Plan estime que les réserves de productivité inexploitées représentent entre 15 et $40 \%$ du niveau présent, formidables enjeux donc!

Quelques années plus tard, les tâches intellectuelles de l'entreprise (activités de conception et de développement) seront aussi touchées par l'introduction de nouvelles méthodes de travail comme la CAO et l'analyse de la valeur qui suppose une bonne aptitude à communiquer dans un travail de groupe et de gestion de l'information

Le dénominateur commun de ces transformations réside dans l'apparition de nouveaux lieux de parole (Cercles de Qualité, groupes de progrès), de nouvelles façons de travailler (travail collectif et d'équipe) et d'une intervention individuelle accrue (enrichissement des tâches, prise de décision). Au cœur de ces activités intellectuelles se trouvent la gestion et la production d'informations.

30 Enfin, élément essentiel pour nous linguistes, les coopérations internationales à l'échelle européenne (Programme ESPRIT, RACE, HERMES, Agence ESA), mais aussi à l'échelle mondiale (dans les TELECOMS, l'espace...) se sont multipliées voire généralisées.

Qu'on se souvienne des laborieuses collaborations franco-anglaises pour le Concorde où le langage des unités n'était même pas normalisé (Plan Metric faisait l'objet d'une campagne d'affichage dans les ateliers anglais en 1975) aujourd'hui un morceau de la tuyère assemblée à la SEP Bordeaux est fabriquée en Italie un autre en Allemagne, Ford Blanquefort reçoit ses pièces des USA et les grands projets spatiaux de l'avenir seront le fruit de collaborations internationales ou ne seront pas du tout tant les coûts sont élevés (la cellule de prospective de l'European Space Agency accueille non seulement des chercheurs venus de tous les pays de la CEE, mais également de toutes les nations ayant une industrie spatiale).

Toutes ces mutations dans les conditions des échanges scientifiques et techniques aboutissent à l'apparition d'une véritable industrie langagière (language business) (Gambier 1990).

33 La CEE compte cinq langues officielles en 1957, neuf depuis 1986, 1 million 5 pages y ont été traduites en 1988 avec une augmentation de 16\%5 en 5 ans la traduction engloutissant $40 \%$ du budget de son fonctionnement des chiffres énormes qui ne sont pas spécifiques à 
la nature de l'institution communautaire une entreprise de Télécommunications à vocation internationale comme Ericsson (Suède) annonce pour 1987 un coût de traduction de $\$ 1$ milliard, 5 pour un chiffre d'affaires de $\$ 5$ milliards.

À la SED, société de communication scientifique, on utilise les images suivantes, les documents fournis au client étranger qui achète notre métro coûtent le prix d'une rame, pour l'exportation d'une centrale nucléaire on traduit 200000 pages, etc.

La multiplication des échanges entre l'université/l'industrie, les donneurs d'ordres/les sous-traitants, les fournisseurs/les clients fait naître une culture d'ouverture sur les autres (cross-culture).

Comprendre les mutations, gérer efficacement nos actions de formation, utiliser les outils nouveaux comme le satellite sont autant de facteurs qui ont profondément changé les conditions de notre intervention.

\section{Le cadavre exquis boira du vin nouveau}

Qui se souvient de la phrase désormais célèbre produite en 1925 par le jeu surréaliste d'association de mots « dictés par la pensée en l'absence de tout contrôle exercé par la raison »? Les enseignants de langue que nous sommes, nous nous situons dans une approche de production langagière diamétralement opposée à celle des surréalistes.

Si nous reconnaissons avec $\mathrm{C}$. Kerbrat-Orecchioni que «l'interaction verbale est la réalité fondamentale du langage»(1986), nous nous plaçons dans une situation sociale caractérisée et déterminée par les relations qui existent ou que l'on veut créer entre les interlocuteurs.

39 C'est ainsi que nous cherchons à encourager des activités d'échanges, de dialogue en introduisant la compréhension du réel et son traitement (comprendre l'existant, et dépasser cette lecture pour « inventer » du sens).

Nous nous efforcerons donc de dépasser la situation de production d'apprentissage qui intervient linéairement et qui appartient à la tradition descriptive (qui ? quoi ? où ? quand?). Ces exercices travaillent davantage la transmission d'informations que la communication.

\section{L'émergence des humanités techniques}

41 Autant de raisons qui nous incitent, pour répondre (répondre, et non nous adapter) à des conditions nouvelles à privilégier la démarche qui consiste à «former à la communication » en langue 1 (le français) et en langue 2 (l'anglais).

Nous ne reviendrons pas sur les acceptions multiples de communication ni dans le débat pour savoir si nous sommes en présence d'un mot clé ou d'un mot omnibus « (A. Moles); nous reprendrons à notre compte les clarifications de R. Escarpit : "La communication est un acte et l'information est son produit » (1976) et de A. Moles : "Ce terme même de " communication « couvre une science nouvelle de "l'interaction" des êtres et des choses " (1986).

43 Dans ces conditions, enseigner la communication à des scientifiques constitue pour l'enseignant-linguiste une "décentration" qui est un fait nouveau en soi puisqu'il s'attarde désormais autant sur la démarche (comment) que sur l'outil (la langue) et autant sur le sujet (l'apprenant) que sur l'objet (produire de l'information et la communiquer en langue étrangère). 
Enfin et c'est là un souci majeur nous nous attachons à prendre en compte l'environnement social dans lequel cet acte de communication se situe. Il est particulièrement éclairant que le $13^{\mathrm{e}}$ congrès du GERAS ait décidé de traiter le thème de la dimension culturelle de l'enseignement des langues à des scientifiques.

Voilà que nous prenons en compte « le bain amniotique dans lequel sont naturellement plongés nos apprenants pour élaborer nos pratiques de formation.

Cette dimension est nouvelle, en dix ans de publication du journal English For Specific Purposes, le lectorat se voit proposer seulement deux articles qui revendiquent une approche culturelle (Jacob 1987 ; Barron 1991), le premier fait référence à la culture de la langue cible et quant au deuxième, il prend en compte la culture technique ancestrale de ses apprenants de Nouvelle-Guinée (Barron 1991).

Il y a donc avec ce congrès reconnaissance implicite de la dimension scientifique et technique des sociétés. Si nous acceptons les néologismes, on peut parler dès lors d'une véritable recentration.

Nous nous situons désormais au cœur des préoccupations et des intérêts de nos apprenants, au niveau de compétence(s) - de connaissances - qui est le leur (point de départ) et avec eux nous progressons - dans le sens d'avancer - afin de favoriser l'information circulante, mais dans le même temps d'agir sur l'information structurante qui vient nécessairement se superposer (synergie du groupe et interaction avec l'animateur-l'enseignant en l'occurrence).

9 Nous intervenons donc à deux niveaux distincts, l'un de surface à savoir la production puis la transmission d'informations, mais dans le même temps et en ce sens nous pouvons parler d'«éducation" à la communication nous mettons en œuvre des processus intellectuels et culturels pour traiter, transformer, le visible, l'immédiatement compréhensible (par ex : discours de Bush, la première étape est « qu'a-t-il annoncé ? », la deuxième est de traiter ces informations à partir de notre situation nationale et locale (Dassault) pour étudier ensemble, les conséquences des réductions du budget de la Défense sur l'industrie de l'armement, le problème des emplois, celui des reconversions, pourquoi est-il si difficile de passer des technologies du militaire au civil...)

Cette démarche revitalise en profondeur la pratique de la communication. Certains diront « enfin!», d'autres «mais est-ce bien raisonnable?» nous sommes tellement peu préparés.

\section{Nouvelle définition de la culture}

51 La dynamique de la reconnaissance par tous de la dimension scientifique et technique (Serres dans le Tiers Instruit, Jacquard dans Inventer l'homme, Levy-Leblond) de la culture d'aujourd'hui n'est pas étrangère à notre propre évolution de linguistes. Nous ne nous inscrivons pas ici dans le débat dépassé sur « la culture cultivée humaniste » opposée à « la culture scientifique et technique ».

52 Ellul écrivait en 1977 que « la pensée technique est radicalement incapable de se penser elle-même. Et par conséquent, elle est incapable de ne traiter aucun problème du monde moderne à sa racine puisque tous ces problèmes ont une racine technique » (1977).

Le démenti a été apporté par les faits récents quand des techniciens comme Testard pour le génie génétique, Jacquard pour la démographie, Cousteau pour l'écologie, Dumont pour 
l'agriculture, ont abandonné leurs paillasses et leur laboratoire pour témoigner, mettre en garde, informer.

Loin de voir là « un coup de bluff », il nous semble que nous assistons à l'élaboration d'un nouveau système de valeur qui place le devenir de l'homme au centre de ses préoccupations.

Pour certains, il y avait urgence de cette " prise de conscience » (awareness) :

Il est impossible de ne pas être frappé de stupeur lorsqu'on constate la fois l'importance concrète des techniques dans la vie des individus et des sociétés et la quasi-absence de réflexion sur ces mêmes techniques de la part de ceux qui font officiellement profession de réfléchir sur la vie des individus et plus généralement des sociétés (Roqueplo 1983)

ou encore le récent plaidoyer de B. d'Espagnat pour « Penser la science » (1990).

Les raisons de cette nouvelle donne et du rôle prééminent que joue désormais la communauté scientifique sont d'ordre divers (développement des sciences, des techniques et prégnance des enjeux dans de nombreux secteurs des vie quotidienne, génie génétique, technologie de l'information, etc.).

57 L'idée largement partagée de la finitude de notre planète confère à chacun d'entre nous une nouvelle citoyenneté et donc de nouvelles responsabilités individuelles et collectives; c'est vrai comme le disait Coluche certains sont plus citoyens que d'autres, le sous-développement excluant des millions d'hommes.

Cette nouvelle citoyenneté exige de pouvoir se déterminer personnellement sur les choix (Testard) (écologie, armement) induits par le développement des recherches et non plus seulement de déléguer les prises de décision à des experts (fussent-ils reconnus par tous pour leur intégrité : Comité d'éthique).

\section{Nouvelle responsabilité des enseignants}

59 Il serait socialement difficile à justifier que l'école et plus généralement le système éducatif tardât à prendre en compte l'émergence de ce qui n'est pas une nouvelle discipline supplantant ou s'ajoutant aux autres, mais que nous définirons plutôt comme une approche différente de la lecture du monde « l'approche interculturelle».

Il y a déjà quelques années F. Gaussen (Le Monde, 15 mai 1984) écrivait :

L'école démocratique de masse qui existe désormais en France doit à la fois définir

la culture que la société entend transmettre et s'adapter à la réalité humaine,

sociale et culturelle des Jeunes qu'elle accueille.

61 Si nous relisons Antoine Bloye de P. Nizan (1985) écrit en 1933 nous comprenons parfaitement quel était alors le rôle de l'école, Antoine, fils d'ouvrier et de femme de ménage, boursier de l'instruction publique se hisse à force d'acharnement à « un poste de commandement", l'école lui a permis de quitter le côté de ceux «qui subissent les ordres »... l'école facteur de « promotion sociale »...

62 Mais aujourd'hui? Ces valeurs ont disparu, remplacées par « une culture de confection relayée par les médias, la culture du scoop, du clinquant, de l'individualisme ; "la culture n'est pas un bien de consommation" dit D. Sallenave (1991).

63 Le temps est venu de nous interroger sur les "contenus" puisque nous disposons de formidables relais médiatiques (culturels ?) à savoir le câble et le satellite (déjà lo\% des lycées câblés, les universités, les entreprises). Des images en continu, des infos non-stop... 

propos aux linguistes enseignant dans les cursus scientifiques, mais bien évidemment la question est posée aux collègues des filières dites classiques ainsi qu'aux historiens et aux géographes (demandeurs de formation en histoire des sciences par exemple), aux enseignants de lettres (qui pour l'instant se "défaussent" le plus souvent de ces responsabilités en les confiant aux CDI et à tous les enseignants y compris aux scientifiques eux-mêmes (qui n'introduisent que rarement la dimension historique à leurs cours et ont tendance à gommer les difficiles accouchements de la science pour n'en présenter que les résultats, ex. : enseignement de la thermodynamique). nécessaire approche interculturelle implique.

sommes issus d'un système fortement marqué par la dichotomie "littéraire/ scientifique» et nous organisons notre relation pédagogique d'enseignant à des scientifiques, dans un rapport culturel ancré (le plus souvent) dans la relation « ignorant/ savant » (où cas remarquable, le non-spécialiste est l'enseignant donc l'ignorant, chose que d'ailleurs certains collègues vivent très mal), mais situation généralement supportée puisque (parce que) le rapport s'inverse quand il s'agit des apprentissages linguistiques et que le formateur retrouve là son statut.

70 Le fait que le discours interculturel ne soit ni formalisé, ni stabilisé puisqu'il se constitue devant nous rend cette démarche encore plus difficile : les connaissances ne peuvent plus être reçues comme définitives, mais comme des étapes provisoires (SIDA et la recherche, les systèmes experts et l'impact sur l'emploi, l'homme dans l'espace ou la multiplication des systèmes automatisés, le génie génétique et la lutte contre les famines, etc.) ces repères qui se déplacent, qui nous échappent constitueront, il ne faut pas se le cacher, 
une composante importante de la problématique pédagogique (au niveau de l'implication des différents acteurs face à un savoir multipolarisé).

\section{Peut-on se préparer à cette prévisible et nécessaire décentration ?}

71 La question nous est posée très concrètement puisque l'ENS de Cachan prépare un groupe de 24 élèves linguistes à enseigner à des scientifiques. Comme Jacquard, j'ai envie de dire, la préparation consiste moins à s'adapter à l'existant qu'à faire preuve d'imagination pour faire autrement.

72 Pour y aider, peut-être pouvons analyser ce qu'induit l'intégration de la culture scientifique et technique, dans notre pédagogie.

73 Si nous avons donc pris le parti de promouvoir la communication en langue étrangère en utilisant ce qui structure la pensée et la parole des apprenants (leur travail, mais également l'individu hors de son travail confronté à des choix portant sur la vie la maladie l'utilisation des découvertes scientifiques, la gestion des ressources, etc.) nous accepterons de nous situer plus dans une situation d'apprentissage que d'enseignement.

Les enjeux sous-tendus par les réponses que l'humanité apportera à ces problèmes sont totalement inédits, l'enseignant encouragera la curiosité, la quête...

75 Il serait illusoire de vouloir se cacher que le débat n'est pas seulement technique, mais aussi politique au sens où il fait apparaître une nouvelle citoyenneté au niveau planétaire. L'enseignant de langue se trouve donc dans la même situation que le collègue historien ou philosophe ou que le citoyen. Il doit pouvoir au-delà du mode d'investigation classique de la description opérer avec ses apprenants à un véritable questionnement des faits, mais aussi des enjeux dont ils sont porteurs.

\section{Pédagogie et décentration}

Comment ne pas engager à beaucoup de prudence vis-à-vis de la «culture de confection " ?J. Hambrook, producteur de matériaux pédagogiques pour l'enseignement des langues raconte volontiers son tournage de "Bid For Power", vidéo destinée à enseigner l'anglais de la négociation dans un contexte international, tout est faux, les trois pays, les situations, il a fabriqué une fiction prétendue authentique.

Pour inscrire notre pratique dans un rapport de transparence avec le réel nous avons recours à la réception par satellite et nous construisons nos enseignements à partir des images reçues. Si nous prenons appui sur une reformulation linéaire des contenus nous nous imposons de promouvoir en toutes circonstances, le dialogue, l'opinion productrice de sens.

Par exemple un reportage de CNN traite d'un accident (Green Run) révélé vingt ans après, du dégagement d'un gaz toxique sur une ville américaine ayant provoqué des morts par cancer; les vents ayant rabattu le nuage destiné aux Russes. Après avoir rassemblé chacun des éléments de l'information (quand, où, comment) nous nous attachons non seulement à retrouver le pourquoi historique (guerre froide) et technique (recherche chimique liée à l'armement), mais aussi à montrer le paradoxe des outils qui interviennent sur le produit en mélangeant le vrai (le nuage, le silence des autorités la mort du cancer tel jour de telle personne), du douteux (lien non prouvé entre les cancers présentés et le nuage).

79 Autre exemple: un an après la guerre du Golf, il est indéniable que la puissance du discours de CNN nous a totalement mystifiés, les débats, discussions, échanges que nous 
avons eu, en cours, s'organisaient autour des idées de guerre technologique de guerre propre, totalement fausse de catastrophe écologique réelle en partie seulement.

Avec le recul, cette manipulation que nous avons relayée nous montre la fragilité de nos propres analyses et la formidable suspicion que nous devons entretenir vis-à-vis de nos sources.

Si la complexité est non pas la clé du monde, mais le défi à affronter, la pensée complexe est non pas ce qui évite ou supprime le défi, mais qui aide à le relever et parfois même à le surmonter. (Morin 1990)

Les concepts que forge E. Morin tout au long de ses entretiens (avec Ardoino par ex) ou dans ses livres (La Méthode, Seuil 1977) ne constituent pas des entités closes, délimitant un champ, un empire, ce sont des concepts ouverts, en boucles, renvoyant toujours à un environnement.

S'il est possible de prendre en compte ces explorations dans la quotidienneté de nos pratiques nous verrons notre rôle davantage comme celui d'un facilitateur dans cette nécessité de faire circuler l'information (orale et écrite) plutôt que comme celui d'un enseignant, la fonction tutoriale devient une fonction secondaire comme réponse individuelle ou collective pour satisfaire la fonction principale.

\section{Conclusion : utopie et avenir}

83 L'école et la société sont perpétuellement en phase de structuration, aucun acquis n'est définitif. Pourquoi les linguistes, favorisés par leur position charnière, n'aideraient-ils pas à l'accouchement des cultures en gestation? Faut-il se résigner à voir dans ce projet interculturel une alternative utopique peu crédible?

Et si pour nous réconcilier autour de cette mobilisation culturelle nous renoncions à parler de culture scientifique et technique pour choisir comme référence la « culture de société » (expression utilisée par D. Jacobi) ?

\section{BIBLIOGRAPHIE}

Barron, C. 1991. « Material thoughts: ESP and culture ». English for Specific Purposes 10/3, 173-187.

Crozier, M. et E. Friedberg. 1977. L'acteur et le système. Paris : Seuil.

D'Espagnat, B. 1990. Penser la Science. Paris : Gauthier-Villars.

Ellul, J. 1977. Le système technicien. Paris : Calmann-Lévy.

Escarpit, R. 1976. Théorie générale de la communication. Paris : Hachette.

Gambier, Y. 1990. «La traduction, nouveaux marchés ». La Pensée, décembre.

Holett, V. 1991. Business Objectives. Oxford : Oxford University Press.

INSEE. 1988. « Enquête sur l'emploi de mars 1988 ». 
Jacques, F. 1979. « Recherches logiques sur le dialogue ». Dialogiques 1, Presses Universitaires de France.

Journal of Business and Technical Communication. 1991. Volume 5/3, July.

Kerbrat-Orecchioni, C. 1986. « Nouvelle communication et analyse conversationnelle ». Langue Française 70, 7-25.

Moles, A. 1986. Théorie structurelle de la communication et société. Paris : Masson.

Morin, E. 1990. Introduction à la pensée complexe. Paris : ESF.

Nizan, P. 1985. Antoine Bloyé, Les Cahiers Rouges. Paris : Grasset.

Ouchy, P. 1980. Z Theory. New York : A. Wesley.

Roqueplo, Ph. 1983. Penser la Technique Paris : Seuil.

Sallenave, D. 1991. « Assouvissement ou émancipation ». Le Monde, 26 sept. 1991.

Serieyx, H. 1982. Mobiliser l'intelligence de l'entreprise. Paris : Entreprise moderne d'édition

Stoffaes, C. 1981. «Informatique et emploi ». Actes du colloque international « Informatique et société ». Documentation française, 29-60.

\section{NOTES}

1. Journal of Business and Technical Communication, Sage Publications. Au sommaire du volume 5/3, 1991, nous notons les articles suivants: Limback, R. «Language skills: Can a value-added approach make a difference? » et Forman, J. « Novices work on group reports: Problems in group writing and in computer supported group writing ».

\section{AUTEUR}

\section{EVELYNE BROUZENG}

Evelyne Brouzeng enseigne au Département de GEII, IUTA, Université de Bordeaux 1. evelyne.brouzeng@wanadoo.fr 